\title{
QUANTUM ERGODICITY FOR RESTRICTIONS TO HYPERSURFACES
}

\author{
SEMYON DYATLOV AND MACIEJ ZWORSKI
}

\begin{abstract}
Quantum ergodicity theorem states that for quantum systems with ergodic classical flows, eigenstates are, in average, uniformly distributed on energy surfaces. We show that if $N$ is a hypersurface in the position space satisfying a simple dynamical condition, the restrictions of eigenstates to $N$ are also quantum ergodic.
\end{abstract}

\section{INTRODUCTION}

In a recent paper [13] Toth and Zelditch proved a remarkable result stating that if $(M, g)$ is a compact manifold with an ergodic geodesic flow, then quantum ergodicity holds for restrictions of eigenfunctions to hypersurfaces satisfying a certain dynamical condition. In an earlier paper [12] they established a similar result for bounded domains in $\mathbb{R}^{n}$ whose boundaries are piecewise smooth and whose billiard flows are ergodic.

The purpose of this note is to provide a short proof of a semiclassical theorem which simultaneously generalizes both results. Our approach avoids global constructions and calculations by reducing equidistribution for restrictions to equidistribution in the ambient manifold. The geometric condition (1.7) enters to obtain a decorrelation between contributions to the restrictions coming from different parts of phase space. The proof uses some ideas of [5, Appendix D] but we do not refer to any results from that paper.

For the standard quantum ergodicity result established by Shnirelman, Zelditch and Colin de Verdière, see [5],[7],[12],[13] and references given there. The case of Riemannian manifolds with piecewise smooth boundaries was established in a special case by Gérard-Leichtnam [6], and by Zelditch and the second author [14] in general. A semiclassical version of quantum ergodicity was first provided by Helffer-MartinezRobert [7].

To make the presentation more clear, in the introduction we will work in the setting of manifolds without boundary, referring to Appendix A for modifications in the case of manifolds with piecewise smooth boundaries.

Let $(M, g)$ be a compact smooth Riemannian manifold and consider

$$
P(h):=-h^{2} \Delta_{g}+V(x), \quad V \in C^{\infty}(M ; \mathbb{R}) .
$$



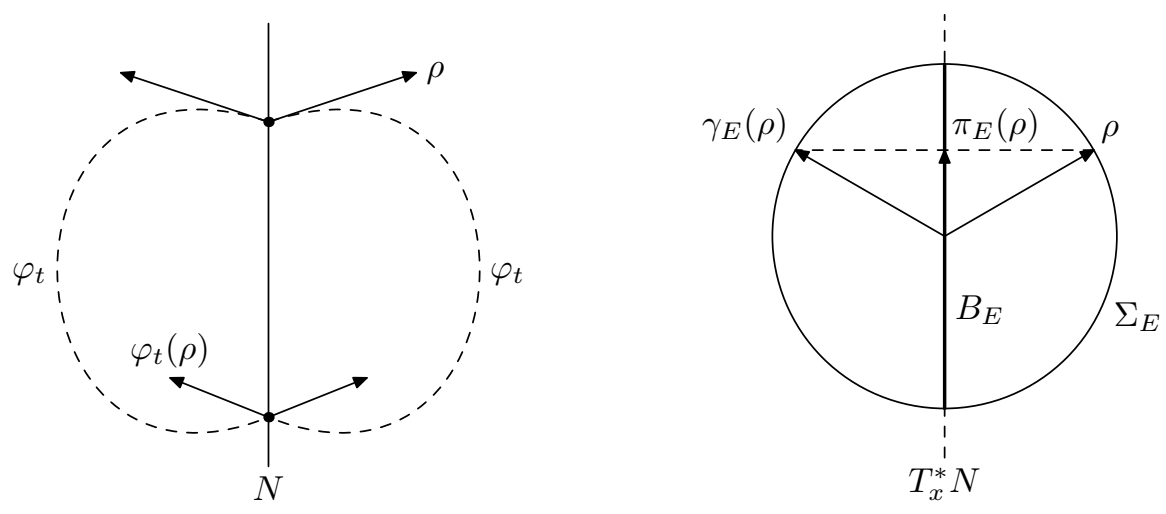

FiguRE 1. Left: the situation prohibited almost everywhere by the dynamical assumption (1.7). Right: The projection map $\pi_{E}$ and the reflection map $\gamma_{E}$ in the cotangent space over some point $x \in N$.

The operator $P(h)$ is self-adjoint when acting on half-densities (see [15, Chapter 9]), $L^{2}\left(M, \Omega_{M}^{1 / 2}\right)$. (This technical point is helpful when more general operators are considered.) The classical symbol of $P(h)$ is given by

$$
p(x, \xi)=|\xi|_{g}^{2}+V(x), \quad(x, \xi) \in T^{*} M,
$$

and $p$ defines the Hamiltonian flow,

$$
\varphi_{t}:=\exp \left(t H_{p}\right): p^{-1}(E) \longrightarrow p^{-1}(E), \quad E \in \mathbb{R},
$$

We make the following assumption on a range on energies:

For $E \in[a, b],\left.d p\right|_{p^{-1}(E)} \neq 0$, and the flow $\varphi_{t}: p^{-1}(E) \rightarrow p^{-1}(E)$ is ergodic, where ergodicity is with respect to the Liouville measure $\mu_{E}$ on $p^{-1}(E)$.

Now, let $N$ be a smooth open hypersurface in $M$. We define

$$
\Sigma_{E}:=p^{-1}(E) \cap \pi^{-1}(N),
$$

where $\pi: T^{*} M \rightarrow M$ is the natural projection. We note that $\Sigma_{E}$ is a smooth hypersurface in $p^{-1}(E)$ if

$$
V(x)=E \Longrightarrow d V(x) \notin N_{x}^{*} N,
$$

and for simplicity we make this assumption for all $E \in[a, b]$. For $E>0$ it is satisfied when $V \equiv 0$, and that is the setting of Theorem 3 .

By restricting elements of $\Sigma_{E}$ to $T N$ we obtain a map

$$
\pi_{E}: \Sigma_{E} \rightarrow B_{E}:=\pi_{E}\left(\Sigma_{E}\right) \subset T^{*} N
$$

which is a local diffeomorphism almost everywhere. It defines a unique nontrivial involution

$$
\gamma_{E}: \Sigma_{E} \rightarrow \Sigma_{E}, \quad \pi_{E} \circ \gamma_{E}=\pi_{E}, \quad \gamma_{E} \circ \gamma_{E}=i d
$$


which is the reflection across the orthogonal complement of the normal bundle $N^{*} N \subset$ $T_{N}^{*} M$ with respect to the metric on the fibers of $T^{*} M$ induced by $g$. This involution enters into the dynamical assumption we make on $N$ (see Fig. 1):

For $E \in[a, b]$, the set of $\rho \in \Sigma_{E}$ satisfying $\varphi_{t}(\rho) \in \Sigma_{E}$

and $\varphi_{t}\left(\gamma_{E}(\rho)\right)=\gamma_{E}\left(\varphi_{t}(\rho)\right)$ for some $t \neq 0$, has measure 0 .

We denote by $u_{j}(h)$ a normalized eigenfunction of $P(h)$ with an eigenvalue $E_{j}(h)$,

$$
\left(P(h)-E_{j}(h)\right) u_{j}(h)=0, \quad\left\|u_{j}(h)\right\|_{L^{2}\left(M, \Omega_{M}^{\frac{1}{2}}\right)}=1 .
$$

To formulate the quantum ergodicity theorem for restrictions, we need to restrict half-densities to $N$ and that requires making a choice. Suppose $f \in C^{\infty}(M),\left.f\right|_{N}=0$, $\left.d f\right|_{N} \neq 0$. Informally, the restriction is now defined using, $|d x|^{\frac{1}{2}}=|d y|^{\frac{1}{2}}|d f|^{\frac{1}{2}}, x \in M$, $y \in N$. More precisely if, in local coordinates, $x=\left(x^{\prime}, x_{n}\right), N=\left\{x_{n}=0\right\}$ then, in the half-density notation of $[15, \S 9.1]$,

$$
\left.\left(u(x)|d x|^{\frac{1}{2}}\right)\right|_{N}:=u\left(x^{\prime}, 0\right)\left|d x^{\prime}\right|^{\frac{1}{2}}\left|\frac{\partial f}{\partial x_{n}}\left(x^{\prime}, 0\right)\right|^{-\frac{1}{2}} .
$$

Using the notation of [15, Chapter 14], reviewed in Section 2 below, we can state our main result. See Appendix A for the modifications needed in the case when $M$ has a boundary.

Theorem 1. Suppose that $(M, g)$ is a compact Riemannian manifold with a piecewise smooth boundary satisfying (A.1) and that $u_{j}=u_{j}(h)$ are normalized eigenfuctions of the Dirichlet realization of $P(h)$.

Suppose also that (1.3) holds and that $N$ is a smooth open hypersurface not intersecting $\partial M$ and satisfying (1.7). For $Q \in \Psi_{h}^{m}\left(M, \Omega_{M}^{1 / 2}\right)$ put $v_{j}:=\left.Q u_{j}(h)\right|_{N}$, where the restriction operator on half densities is defined in (1.8). Then for $A \in \Psi_{h}^{0}\left(N, \Omega_{N}^{1 / 2}\right)$, compactly supported in $N$, we have

$$
\left.h^{n} \sum_{E_{j} \in[a, b]}\left|\left\langle A v_{j}, v_{j}\right\rangle_{L^{2}\left(N, \Omega_{N}^{1 / 2}\right)}-\int_{\Sigma_{E_{j}}} \pi_{E_{j}}^{*} \sigma(A)\right| \sigma(Q)\right|^{2} d \nu_{E_{j}} \mid \longrightarrow 0, \quad h \rightarrow 0,
$$

where $\sigma(A) \in S^{0}\left(T^{*} N\right)$ is the symbol of $A, \sigma(Q) \in S^{m}\left(T^{*} M\right)$ is the symbol of $Q$, and the measure $\nu_{E}$ is defined on $\Sigma_{E}$ by the identity

$$
\frac{d \mu_{E}}{\mu_{E}\left(p^{-1}(E)\right)}=d \nu_{E} \wedge d f
$$

with $\mu_{E}$ the Liouville measure and $f$ defining the restriction of half-densities in (1.8).

Remarks. (i) The measure $\nu_{E}$ has a particularly nice description in the case $V=0$. Assume that $E=1$, then $\Sigma_{1}=S_{N}^{*} M$ where $S_{N}^{*} M \subset S^{*} M$ denotes the cosphere bundle 
of $M$ restricted to $N$. The Liouville measure, $\mu_{1}$, on $S^{*} M$, induces, for each $x \in M$, a measure on $S_{x}^{*} M, \mu_{x}$, such that

$$
\mu_{1}(\Omega)=\int_{M} \mu_{x}\left(\Omega \cap S_{x}^{*} M\right) d \operatorname{vol}_{g}(x), \quad \Omega \subset S^{*} M .
$$

Our measure on $\Sigma_{1}=S_{N}^{*} M$ is then given by

$$
\nu_{1}(\Gamma)=\frac{1}{\mu_{1}\left(S^{*} M\right)} \int_{N} \mu_{x}\left(\Gamma \cap S_{x}^{*} M\right) d \operatorname{vol}_{\left.g\right|_{N}}(x), \quad \Gamma \subset S_{N}^{*} M,
$$

where $\left.g\right|_{N}$ is the metric on $N$ induced by $g$. (Here $\Omega$ and $\Gamma$ are Borel sets.) See Appendix B for details.

(ii) The now standard argument due to Colin de Verdière and Zelditch and described in [15, Theorem 15.5] shows that this result provides pointwise convergence for a density one subsequence: there exists a family of sets, $\Lambda(h) \subset\left\{a \leq E_{j} \leq b\right\}$, such that $\lim _{h \rightarrow 0} \frac{\# \Lambda(h)}{\#\left\{a \leq E_{j} \leq b\right\}}=1$, and, in the notation of Theorem 1 ,

$$
\left.\sup _{E_{j} \in \Lambda(h)}\left|\left\langle A v_{j}, v_{j}\right\rangle_{L^{2}\left(N, \Omega_{N}^{1 / 2}\right)}-\int_{\Sigma_{E_{j}}} \pi_{E_{j}}^{*} \sigma(A)\right| \sigma(Q)\right|^{2} d \nu_{E_{j}} \mid \longrightarrow 0, \quad \text { as } h \rightarrow 0 .
$$

In step 4 of the proof of [15, Theorem 15.5], to pass from (1.12) for a countable dense family $A_{k}$ of pseudodifferential operators to the full statement, one needs uniform boundedness of $v_{j}$ on $L^{2}(N)$. While this need not be true for the whole sequence, it holds for $E_{j} \in \Lambda(h)$ if we take one of the operators $A_{k}$ to be the identity.

(iii) The dynamical condition of Toth-Zelditch [13] is stated using Poincaré return times but the analysis in that paper shows that it is equivalent to our condition (1.7). The paper [13] provides interesting examples for which it is satisfied.

(iv) We sum over eigenvalues in a fixed size interval $[a, b]$ in (1.9) since the corresponding smoothed out spectral projectors are pseudodifferential operators. It would be interesting to prove an analogous statement for size $h$ intervals, whose projectors have more complicated microlocal structure, as in [5, Appendix D].

The paper is organized as follows. In Section 2 we review basic concepts of semiclassical quantization and present slightly non-standard facts needed in the proof. The key point is that even in the case of manifolds with boundary we only need to work with standard pseudodifferential operators. In Section 3 we present a general decorrelation result and in Section 4 the proof of Theorem 1. Since no reference for quantum ergodicity for semiclassical boundary value problems seems to be available, Appendix A present a proof in the spirit of [14] with some simplifications based on [5, Appendix D] (see Lemma 2.2). The high energy result for Laplacians, as presented in [13], follows from Theorem 1 but there is an issue at energy 0, and Appendix B shows how that is overcome. 
Except for the efficiency provided by direct semiclassical methods [15], the proofs are similar to those in [13]. The one significant difference is the treatment of quantum ergodicity for microlocal Cauchy data in Section 4 - see [3] for comparison.

Acknowledgements. We would like to thank Nicolas Burq, Oran Gannot and Stéphane Nonnenmacher for helpful comments on the first version of this paper, Hart Smith for explaining an alternative proof of Lemma 2.1 based on finite speed of propagation, Steve Zelditch for encouraging us to handle the boundary case, and an anonymous referee for many helpful suggestions. The partial support by National Science Foundation under the grant DMS-1201417 is also gratefully acknowledged.

\section{Semiclassical PRELIMINARIES}

We will use the calculus of semiclassical pseudodifferential operators described in $[15$, $\S 9.3, \S 14.2]$. Our operators will always be supported away from the boundary of the manifold and hence can be considered as operators on a boundaryless manifold (for example, by considering the double space of a neighborhood of our manifold in a slightly bigger open manifold without boundary). A notable exception is the Schrödinger operator $-h^{2} \Delta_{g}+V(x)$, which can however be extended smoothly past the boundary.

For a compact manifold, $X$ (which could be different from the compact manifold $M$ considered in Section 1), the class $\Psi_{h}^{m}(X)$ denotes operators of order $m$, so that, for instance $-h^{2} \Delta_{g} \in \Psi_{h}^{2}(M)$. We have the symbol map, $\sigma$, appearing in the following exact sequence

$$
0 \longrightarrow h \Psi_{h}^{m-1}(X) \longrightarrow \Psi_{h}^{m}(X) \stackrel{\sigma}{\longrightarrow} S^{m}\left(T^{*} X\right) / h S^{m-1}\left(T^{*} X\right) \longrightarrow 0,
$$

where $S^{m}$ denotes the standard space of symbols. We take some quantization map $\mathrm{Op}_{h}: S^{m}\left(T^{*} X\right) \rightarrow \Psi_{h}^{m} ;$ it satisfies

$$
\sigma\left(\mathrm{Op}_{h}(a)\right)=a \bmod h S^{m-1}\left(T^{*} X\right) .
$$

We also introduce the class of compactly microlocalized pseudodifferential operators, $\Psi_{h}^{\mathrm{comp}}(X): A \in \Psi^{-\infty}(X)$ is in $\Psi_{h}^{\mathrm{comp}}(X)$ if for some $\chi \in C_{\mathrm{c}}^{\infty}\left(T^{*} X\right)$,

$$
\mathrm{Op}_{h}(1-\chi) A \in h^{\infty} \Psi^{-\infty}(X) \text {. }
$$

For this class the definition of $\mathrm{WF}_{h}(A)$ given in $[15, \S 8.4]$ applies. From the same section we take the definition of microlocal equality of operators.

Following [4, §2.3], [10, §3], and [15, §11.2] we consider Fourier integral operators quantizing a canonical transformation $\kappa: U_{1} \rightarrow U_{2}, U_{1} \Subset T^{*} X$ and $U_{2} \Subset T^{*} Y, \kappa$ defined on a neighbourhood of $U_{1}$ : we say that an operator $F: L^{2}(X) \rightarrow L^{2}(Y)$, quantizes $\kappa$ if for any $A \in \Psi_{h}^{\text {comp }}(Y)$ with $\mathrm{WF}_{h}(A) \Subset U_{2}$,

$$
F^{*} A F=B, \quad B \in \Psi_{h}^{\mathrm{comp}}(X), \quad \sigma(B)=\kappa^{*} \sigma(A) .
$$


We further require that $F$ be microlocally unitary in the sense that $F^{-1}=F^{*}$ microlocally near $U_{1} \times U_{2}$. If $F$ quantizes $\kappa$, then the operator $F^{*}$ quantizes $\kappa^{-1}$. More generally we say that $G$ is a Fourier integral operator associated to the canonical relation $\kappa$ if $G=A F$, for $F$ above and some $A \in \Psi_{h}^{0}(X)$.

The standard example is given by $F(t)=e^{-i t P(h) / h}$, where $P(h)=-h^{2} \Delta_{g}+V(x) \in$ $\Psi_{h}^{2}(M)$ (or a more general operator) which quantizes the Hamiltonian flow $\varphi_{t}:=$ $\exp \left(t H_{p}\right)$.

We say that a tempered operator (see $[15, \S 8.4]) G: L^{2}(X) \rightarrow L^{2}(Y)$, is compactly microlocalized if for some $A \in \Psi_{h}^{\text {comp }}(Y)$ and $B \in \Psi_{h}^{\text {comp }}(X)$,

$$
A G B-G \in h^{\infty} \Psi^{-\infty} \text {. }
$$

In that case we can define $\mathrm{WF}_{h}^{\prime}(G) \subset T^{*} Y \times T^{*} X$, by taking the twisted $\mathrm{WF}_{h}$ of its Schwartz kernel, $K_{G}$ :

$$
\mathrm{WF}_{h}^{\prime}(G):=\left\{(y, \eta ; x,-\xi):(y, \eta ; x, \xi) \in \mathrm{WF}_{h}\left(K_{G}\right)\right\} .
$$

If $F$ is associated to some canonical transformation $\kappa$, then $\mathrm{WF}_{h}^{\prime}(F)$ lies inside the graph of $\kappa$.

We recall from [15, Theorem 14.9] that if $M$ has no boundary, and $P(h)=-h^{2} \Delta+$ $V(x), f \in C_{\mathrm{c}}^{\infty}(\mathbb{R})$, then

$$
f(P(h)) \in \Psi_{h}^{\mathrm{comp}}(M), \quad \sigma(f(P(h)))=f(p) .
$$

We now present three lemmas which will be used in the paper. We assume that the manifold $M$ and operator $P(h)$ are as in Appendix A.

The first lemma makes an observation that $f(P)$ is a nice operator away from the boundary.

Lemma 2.1. Suppose that $f \in C_{\mathrm{c}}^{\infty}(\mathbb{R}), \chi \in C_{\mathrm{c}}^{\infty}\left(M^{\circ}\right)$. Then

$$
f(P(h)) \chi=A_{f}+\mathcal{O}_{L^{2}(M) \rightarrow L^{2}(M)}\left(h^{\infty}\right),
$$

where $A_{f} \in \Psi_{h}^{\mathrm{comp}}\left(M^{\circ}\right)$ is compactly supported away from the boundary and its principal symbol is given by $f(p(x, \xi)) \chi(x)$.

Proof. Let $\psi_{0} \in C_{\mathrm{c}}^{\infty}((-\delta, \delta))$ be equal to 1 near 0 . We write $f(P) \chi$ using the Schrödinger propagator,

$f(P) \chi=\frac{1}{2 \pi h} \int_{\mathbb{R}} \hat{f}(t / h) e^{i t P / h} \chi d t=\frac{1}{2 \pi h} \int_{\mathbb{R}} \hat{f}(t / h) \psi_{0}(t) e^{i t P / h} \chi d t+\mathcal{O}_{L^{2}(M) \rightarrow L^{2}(M)}\left(h^{\infty}\right)$,

where the error estimate follows from the decay of $\hat{f}$ and the untarity of $e^{i t P / h}$. We then write

$$
f(P) \chi=\frac{1}{2 \pi h} \int_{\mathbb{R}} \int_{\mathbb{R}} f(\tau) e^{\frac{i t}{h}(P-\tau)} \chi \psi_{0}(t) d t d \tau .
$$


Choose $\delta$ sufficiently small so that for $|t| \leq \delta, p(x, \xi) \in \operatorname{supp} f$, and $x \in \operatorname{supp} \chi$, we have $d_{g}\left(\pi\left(\varphi_{t}(x, \xi)\right), \partial M\right)>\epsilon>0$. In that case we can use the local parametrix for $e^{i t P / h}$, see for instance $[15, \S 10.2]$. An application of the stationary phase method in $(t, \tau)$ variables gives the conclusion of the lemma.

As before, let $\left(u_{j}(h)\right)_{j \in \mathbb{N}}$ be the full orthonormal system of eigenfunctions of $P(h)$ with eigenvalues $E_{j}(h)$. Lemma 2.1 applied to the operator $f(P(h)) A$, where $A \in$ $\Psi_{h}^{m}(M)$ is supported away from the boundary, and $f \in C_{\mathrm{c}}^{\infty}(\mathbb{R})$, gives, together with the trace formula [15, Theorem 14.10]

$$
(2 \pi h)^{n} \sum_{j} f\left(E_{j}\right)\left\langle A u_{j}, u_{j}\right\rangle=\int_{T^{*} M} f(p) \sigma(A) d \mu_{\sigma}+\mathcal{O}(h),
$$

where $\mu_{\sigma}$ is the symplectic measure, $\mu_{\sigma}=\sigma^{n} / n$ !.

The second lemma, in the spirit of [5, Appendix D], gives estimates using $L^{2}$ norms of symbols:

Lemma 2.2. Suppose that $A \in \Psi_{h}^{m}\left(M^{\circ}\right)$ is a pseudodifferential operator compactly supported away from $\partial M$. Then for each $a^{\prime}<a<b<b^{\prime}$,

$$
(2 \pi h)^{n} \sum_{E_{j} \in[a, b]}\left\|A u_{j}\right\|_{L^{2}}^{2} \leq\|\sigma(A)\|_{L^{2}\left(p^{-1}\left(\left[a^{\prime}, b^{\prime}\right]\right)\right)}^{2}+\mathcal{O}(h)
$$

where the $L^{2}$ norm of $\sigma(A)$ is taken with respect to the measure $\mu_{\sigma}$.

More generally, if $N \subset M$ is a fixed smooth submanifold (of any dimension) not intersecting $\partial M$, then there exists a constant $C$ such that for each $\widetilde{A} \in \Psi_{h}^{m}(N)$ supported in a fixed compact subset of $N$,

$$
h^{n} \sum_{E_{j} \in[a, b]}\left\|\widetilde{A}\left(\left.u_{j}\right|_{N}\right)\right\|_{L^{2}}^{2} \leq C\|\sigma(\widetilde{A})\|_{L^{2}\left(\pi\left(p^{-1}\left(\left[a^{\prime}, b^{\prime}\right]\right) \cap T_{N}^{*} M\right)\right)}^{2}+\mathcal{O}(h) .
$$

Here $T_{N}^{*} M$ is the cotangent bundle of $M$ restricted to $N$ and $\pi: T_{N}^{*} M \rightarrow T^{*} N$ is the projection.

Remark. We note that in the case when $\widetilde{A}=1$ we recover the bound

$$
h^{n} \sum_{E_{j} \in[a, b]}\left\|\left.u_{j}\right|_{N}\right\|_{L^{2}}^{2} \leq C .
$$

By contrast, for individual eigenfuctions the bound $C h^{\frac{n-k}{2}}$ is optimal - see [1] and [11]. 
Proof. To show (2.7), take $f \in C_{\mathrm{c}}^{\infty}\left(a^{\prime}, b^{\prime}\right)$ such that $0 \leq f \leq 1$ everywhere and $f=1$ on $[a, b]$. Then we write by $(2.6)$,

$$
\begin{gathered}
(2 \pi h)^{n} \sum_{E_{j} \in[a, b]}\left\|A u_{j}\right\|_{L^{2}}^{2} \leq(2 \pi h)^{n} \sum_{j} f\left(E_{j}\right)\left\langle A^{*} A u_{j}, u_{j}\right\rangle \\
=\int_{T^{*} M} f(p)|\sigma(A)|^{2} d \mu_{\sigma}+\mathcal{O}(h) \leq \int_{p^{-1}\left(\left[a^{\prime}, b^{\prime}\right]\right)}|\sigma(A)|^{2} d \mu_{\sigma}+\mathcal{O}(h) .
\end{gathered}
$$

To show (2.8), denote by $R_{N}: C^{\infty}(M) \rightarrow C^{\infty}(N)$ the restriction operator and note that

$$
\begin{aligned}
h^{n} \sum_{E_{j} \in[a, b]}\left\|\widetilde{A}\left(\left.u_{j}\right|_{N}\right)\right\|_{L^{2}}^{2} & \leq h^{n} \sum_{j}\left|f\left(E_{j}\right)\right|^{2}\left\|\widetilde{A} R_{N} u_{j}\right\|_{L^{2}}^{2}=h^{n} \sum_{j}\left\|\widetilde{A} R_{N} f(P) u_{j}\right\|_{L^{2}}^{2} \\
& =h^{n}\left\|\widetilde{A} R_{N} f(P)\right\|_{\mathrm{HS}}^{2} .
\end{aligned}
$$

The Hilbert-Schmidt norm on the right-hand side is equal to the $L^{2}$ norm of the Schwartz kernel $K$ of $\widetilde{A} R_{N} f(P)$. Recall that $f(P)$ is a pseudodifferential operator when localized away from the boundary, by Lemma 2.1. Note that $K=\mathcal{O}\left(h^{\infty}\right)$ away from the diagonal of $N$ embedded in $N \times M$. To estimate $K$ near the diagonal, we choose local coordinates $x=\left(x^{\prime}, x^{\prime \prime}\right), x^{\prime} \in \mathbb{R}^{k}, x^{\prime \prime} \in \mathbb{R}^{n-k}$, where $k=\operatorname{dim} N$, on $M$ near some point of $N$, in which $N$ is given by $\left\{x^{\prime \prime}=0\right\}$. If $\tilde{a}$ is the full symbol of $\widetilde{A}$ in these coordinates (in the standard quantization) and $\tilde{b}$ is the full symbol of the pseudodifferential operator $f(P(h))$, then we can write

$$
K(x, y)=(2 \pi h)^{-n-k} \int e^{\frac{i}{h}\left(x \cdot \eta-z \cdot \eta+z \cdot \xi^{\prime}-y \cdot \xi\right)} \tilde{a}(x, \eta) \tilde{b}(z, 0, \xi) d z d \eta d \xi
$$

here $y, \xi \in \mathbb{R}^{n}$ and $x, z, \eta \in \mathbb{R}^{k}$. By the unitarity of the (semiclassical) Fourier transform, the $L_{x, y}^{2}$ norm of $K(x, y)$ is equal to the $L_{x, \xi}^{2}$ norm of

$$
K_{1}(x, \xi)=(2 \pi h)^{-n / 2-k} \int e^{\frac{i}{h}\left(x \cdot \eta-z \cdot \eta+z \cdot \xi^{\prime}\right)} \tilde{a}(x, \eta) \tilde{b}(z, 0, \xi) d z d \eta .
$$

The method of stationary phase shows that

$$
K_{1}(x, \xi)=(2 \pi h)^{-n / 2} e^{\frac{i}{h} x \cdot \xi^{\prime}}\left(\tilde{a}\left(x, \xi^{\prime}\right) \tilde{b}(x, 0, \xi)+\mathcal{O}_{C^{\infty}}(h)\right) .
$$

Now, $h^{n / 2}$ times the $L^{2}$ norm of $K_{1}$ is bounded by a constant times the $L^{2}$ norm of $\tilde{a}$ on the set $\pi\left(\operatorname{supp} \tilde{b} \cap T_{N}^{*} M\right)$, with an $\mathcal{O}(h)$ remainder.

To formulate the next lemma we define

$$
\operatorname{Diag}\left(T^{*} M\right):=\left\{(\rho, \rho): \rho \in T^{*} M\right\} \subset T^{*} M \times T^{*} M .
$$

Lemma 2.3. Suppose that $G: L^{2}(M) \rightarrow L^{2}(M)$ is a compactly microlocalized tempered operator in the sense of (2.2), compactly supported away from the boundary, and that $f \in C_{\mathrm{c}}^{\infty}(\mathbb{R})$. Then for $G$ satisfying

$$
\mathrm{WF}_{h}^{\prime}(G) \cap \operatorname{Diag}\left(T^{*} M\right)=\emptyset,
$$


we have

$$
\sum_{j} f\left(E_{j}\right)\left\langle G u_{j}, u_{j}\right\rangle=\mathcal{O}\left(h^{\infty}\right)
$$

Proof. The left-hand side of (2.10) is equal to the trace of $G f(P(h))$. We can write $G$ as a finite sum of operators of the form $X_{1} G X_{2}$, where $X_{1}, X_{2} \in \Psi_{h}^{\text {comp }}$ satisfy $\mathrm{WF}_{h}\left(X_{1}\right) \cap \mathrm{WF}_{h}\left(X_{2}\right)=\emptyset$. Then by the cyclicity of the trace,

$$
\operatorname{Tr}\left(X_{1} G X_{2} f(P)\right)=\operatorname{Tr}\left(X_{2} f(P) X_{1} G\right)=\mathcal{O}\left(h^{\infty}\right),
$$

as $X_{2} f(P) X_{1} \in h^{\infty} \Psi^{-\infty}$, by Lemma 2.1.

\section{Decorrelation for Fourier integral operators}

In the proof of Theorem 1 we will encounter expressions involving $\left\langle F u_{j}, u_{j}\right\rangle$, where $\left(u_{j}(h)\right)_{j \in \mathbb{N}}$ is the full orthonormal system of eigenfunctions of $P(h)=-h^{2} \Delta_{g}+V(x)$ with eigenvalues $E_{j}(h)$, and $F$ is a compactly microlocalized semiclassical Fourier integral operator. This section shows that the sum of such terms over $j$ in an $\mathcal{O}(1)$ sized spectral window is negligible when the canonical relation of $F$ satisfies a 'nonreturning' assumption; we call this phenomenon decorrelation for Fourier integral operators.

Assume $F$ is a compactly microlocalized tempered operator $L^{2}(M) \rightarrow L^{2}(M)$, in the sense of (2.2), and

$$
\|F\|_{L^{2} \rightarrow L^{2}}=\mathcal{O}(1), \mathrm{WF}_{h}^{\prime}(F) \subset\left\{(\kappa(\rho), \rho): \rho \in K_{1}\right\},
$$

where the wavefront set is defined in (2.3), $\kappa: V_{1} \rightarrow V_{2}$ is a canonical transformation, $V_{1}, V_{2} \subset T^{*} M$ are open sets, and $K_{j} \subset V_{j}$ are compact sets such that $\kappa\left(K_{1}\right)=K_{2}$. (In our case, $F$ will be a Fourier integral operator, but this is not required in the proof.)

For each $t \in \mathbb{R}$, define the $t$-exceptional set,

$$
\mathcal{E}_{\kappa}(t):=\left\{\rho \in K_{1} \cap \varphi_{-t}\left(K_{1}\right): \varphi_{t}(\kappa(\rho))=\kappa\left(\varphi_{t}(\rho)\right)\right\}, \quad \varphi_{t}:=\exp \left(t H_{p}\right) .
$$

Here $\varphi_{t}$ is the broken Hamiltonian flow of $p$, defined almost everywhere and described in Appendix A.

The decorrelation result is given as follows:

Lemma 3.1. Suppose that $a<b$ are fixed, and that there exists $t_{0}>0$ such that

$$
\mu_{\sigma}\left(p^{-1}([a, b]) \cap \bigcup_{|t| \geq t_{0}} \mathcal{E}_{\kappa}(t)\right)=0,
$$

where $\mathcal{E}_{\kappa}(t)$ is given by (3.2) and $\mu_{\sigma}$ is the symplectic measure.

Then for each $F$ compactly supported inside $M^{\circ}$ and satisfying (3.1),

$$
h^{n} \sum_{E_{j} \in[a, b]}\left|\left\langle F u_{j}, u_{j}\right\rangle\right| \rightarrow 0 \text { as } h \rightarrow 0 .
$$


Proof. Take $T>t_{0}$ and denote

$$
\widetilde{K}_{T}:=\left(\left(\mathcal{B}_{T} \cup \kappa^{-1}\left(\mathcal{B}_{T}\right)\right) \cap K_{1}\right) \cup \bigcup_{t_{0} \leq|t| \leq T} \mathcal{E}_{\kappa}(t) .
$$

Here $\mathcal{B}_{T}$ is the set of points at which the broken Hamiltonian flow is not well-defined on the interval $[-T, T]$, see (A.2). Then $\widetilde{K}_{T}$ is a compact subset of $U_{1}$ and $\mu_{\sigma}\left(\widetilde{K}_{T} \cap\right.$ $\left.p^{-1}([a, b])\right)=0$. Therefore, there exists an open set $\widetilde{U}_{T} \subset U_{1}$ and constants $a^{\prime}<a$ and $b^{\prime}>b$ such that

$$
\widetilde{K}_{T} \subset \widetilde{U}_{T}, \quad \mu_{\sigma}\left(\widetilde{U}_{T} \cap p^{-1}\left(\left[a^{\prime}, b^{\prime}\right]\right)\right) \leq T^{-1} .
$$

Take $X_{T} \in \Psi_{h}^{\mathrm{comp}}(M)$ satisfying $\left|\sigma\left(X_{T}\right)\right| \leq 1, \mathrm{WF}_{h}\left(X_{T}\right) \subset \widetilde{U}_{T}$, and $X_{T}=1 \mathrm{mi}-$ crolocally near $\widetilde{K}_{T}$. Since $F$ is bounded on $L^{2}(M),\left|\left\langle F X_{T} u_{j}, u_{j}\right\rangle\right| \leq C\left\|X_{T} u_{j}\right\|_{L^{2}}$. Hence (2.7) and (A.3) give

$$
\begin{gathered}
h^{n} \sum_{E_{j} \in[a, b]}\left|\left\langle F X_{T} u_{j}, u_{j}\right\rangle\right| \leq C\left(h^{n} \sum_{E_{j} \in[a, b]}\left\|X_{T} u_{j}\right\|_{L^{2}}^{2}\right)^{\frac{1}{2}} \\
\leq C\left(\left\|\sigma\left(X_{T}\right)\right\|_{L^{2}\left(p^{-1}\left(\left[a^{\prime}, b^{\prime}\right]\right)\right)}^{2}+\mathcal{O}_{T}(h)\right)^{\frac{1}{2}} \leq C\left(T^{-1}+\mathcal{O}_{T}(h)\right)^{\frac{1}{2}},
\end{gathered}
$$

where $C$ denotes a constant independent of $T$ and $h$.

We now analyse the contribution of $F_{1}:=F\left(1-X_{T}\right)$. For that define

$$
\left\langle F_{1}\right\rangle_{T}:=\frac{1}{T} \int_{0}^{T} e^{i t P(h) / h} F_{1} e^{-i t P(h) / h} d t .
$$

For each eigenfunction $u_{j}$, we have

$$
\left\langle F_{1} u_{j}, u_{j}\right\rangle=\left\langle\left\langle F_{1}\right\rangle_{T} u_{j}, u_{j}\right\rangle .
$$

We now take some $f \in C_{\mathrm{c}}^{\infty}(\mathbb{R})$ such that $0 \leq f \leq 1$ everywhere and $f=1$ near $[a, b]$. Then by (A.3),

$$
\begin{gathered}
h^{n} \sum_{E_{j} \in[a, b]}\left|\left\langle F_{1} u_{j}, u_{j}\right\rangle\right|=h^{n} \sum_{E_{j} \in[a, b]}\left|\left\langle\left\langle F_{1}\right\rangle_{T} u_{j}, u_{j}\right\rangle\right| \\
\leq C\left(h^{n} \sum_{j} f\left(E_{j}\right)\left\|\left\langle F_{1}\right\rangle_{T} u_{j}\right\|_{L^{2}}^{2}\right)^{\frac{1}{2}} .
\end{gathered}
$$

We now write

$$
\begin{gathered}
h^{n} \sum_{j} f\left(E_{j}\right)\left\|\left\langle F_{1}\right\rangle_{T} u_{j}\right\|_{L^{2}}^{2}=h^{n} \sum_{j} f\left(E_{j}\right)\left\langle\left\langle F_{1}\right\rangle_{T}^{*}\left\langle F_{1}\right\rangle_{T} u_{j}, u_{j}\right\rangle \\
=\frac{1}{T^{2}} \int_{0}^{T} \int_{0}^{T} h^{n} \sum_{j} f\left(E_{j}\right)\left\langle e^{i(s-t) P(h) / h} F_{1}^{*} e^{i(t-s) P(h) / h} F_{1} u_{j}, u_{j}\right\rangle d t d s .
\end{gathered}
$$


Since $\left\|e^{i(s-t) P(h) / h} F_{1}^{*} e^{i(t-s) P(h) / h} F_{1}\right\|_{L^{2} \rightarrow L^{2}}$ is bounded uniformly in $t, s, h$, we estimate the integral over the region $|t-s| \leq t_{0}$ using the upper bound on the number of eigenvalues, (A.3),

$$
\frac{1}{T^{2}} \int_{\substack{0 \leq t, s \leq T \\|t-s| \leq t_{0}}} h^{n} \sum_{j} f\left(E_{j}\right)\left\langle e^{i(s-t) P(h) / h} F_{1}^{*} e^{i(t-s) P(h) / h} F_{1} u_{j}, u_{j}\right\rangle d t d s \leq C T^{-1},
$$

where $C$ is again a constant independent of $T$ and $h$.

It remains to estimate the integral over the region $t_{0} \leq|t-s| \leq T$. For $t_{0} \leq|r| \leq T$, define the operator $G_{r}=e^{i r P(h) / h} F_{1}^{*} e^{-i r P(h) / h} F_{1}$, and rewrite the studied integral as

$$
\frac{1}{T^{2}} \int_{\substack{0 \leq t, s \leq T \\|t-s| \geq t_{0}}} h^{n} \sum_{j} f\left(E_{j}\right)\left\langle G_{s-t} u_{j}, u_{j}\right\rangle d t d s .
$$

Take $\chi \in C_{c}^{\infty}\left(M^{\circ}\right)$ such that $F_{1}=F_{1} \chi$, and thus $G_{r}=G_{r} \chi$, then by the cyclicity of the trace as in the proof of Lemma 2.3, we can replace $G_{s-t}$ by $\chi G_{s-t}$ with an $\mathcal{O}_{T}\left(h^{\infty}\right)$ penalty. However, by Lemma A.1, for $t_{0} \leq|r| \leq T$,

$$
\mathrm{WF}_{h}^{\prime}\left(\chi G_{r}\right) \subset\left\{\left(\rho^{\prime}, \rho\right): \rho \in K_{1} \backslash \widetilde{K}_{T}, \varphi_{r}\left(\rho^{\prime}\right) \in K_{1}, \kappa\left(\varphi_{r}\left(\rho^{\prime}\right)\right)=\varphi_{r}(\kappa(\rho))\right\} .
$$

The definition of $\widetilde{K}_{T}$ - see (3.2) and (3.5) - shows that the set in (3.9) does not intersect $\operatorname{Diag}\left(T^{*} M\right)$. This means that the operator $\chi G_{r}$ satisfies the hypothesis of Lemma 2.3, and by (2.10) we find

$$
\frac{1}{T^{2}} \iint_{\substack{0 \leq t, s \leq T \\|t-s| \geq t_{0}}} h^{n} \sum_{j} f\left(E_{j}\right)\left\langle\chi G_{s-t} u_{j}, u_{j}\right\rangle d t d s=\mathcal{O}_{T}\left(h^{\infty}\right) .
$$

Combining this with (3.8) and recalling (3.6) and (3.7), we get

$$
h^{n} \sum_{E_{j} \in[a, b]}\left|\left\langle F u_{j}, u_{j}\right\rangle\right| \leq\left(C T^{-1}+\mathcal{O}_{T}(h)\right)^{\frac{1}{2}}
$$

where $C$ is a constant independent of $T$ and $h$. By choosing $T$ large and then $h$ small, we obtain (3.4).

\section{QUANTUM ERGODICITY FOR RESTRICTIONS}

We will now prove Theorem 1 and we use the notation from the second (semiclassical) part of Section 1. To simplify the presentation we put $Q=i d$. The general case is similar.

We start with some geometric observations. The condition (1.5) shows that, in the notation of (1.6), $B_{E}:=\pi_{E}\left(\Sigma_{E}\right) \subset T^{*} N$, is a smooth manifold with a smooth boundary. Any $\rho \in B_{E} \backslash \partial B_{E}$ is a regular value of $\pi_{E}$; moreover, $\pi_{E}^{-1}(\rho)=\left\{\rho_{+}, \rho_{-}\right\}$, $\pi_{E}$ is a local diffeomorphism near $\rho_{ \pm}$, and the involution $\gamma_{E}$ is given by $\gamma_{E}\left(\rho_{ \pm}\right)=\rho_{\mp}$. The Hamilton vector field $H_{p}$ is transversal to $\Sigma_{E}$ at $\rho_{ \pm}$. 
To prove Theorem 1 we can assume that $[a, b]$ is a small neighbourhood of a fixed energy level $E$. We then decompose any compactly supported $A \in \Psi_{h}^{0}(N)$ as follows:

$$
A=\sum_{j=1}^{J} \widetilde{A}_{j, \epsilon}+A_{\epsilon}+\left(1-X_{E}\right) A
$$

where

- $X_{E} \in \Psi_{h}^{\text {comp }}(N)$ is microlocally equal to $i d$ near $B_{E} \subset T^{*} N$, and $\mathrm{WF}_{h}\left(X_{E}\right)$ is contained in small neighbourhood of $B_{E}$,

- $\widetilde{A}_{j, \epsilon} \in \Psi_{h}^{\mathrm{comp}}(N)$, and $\mathrm{WF}_{h}\left(\widetilde{A}_{j, \epsilon}\right)$ is a small open subset of $B_{E} \backslash \partial B_{E}$,

- $A_{\epsilon} \in \Psi_{h}^{\mathrm{comp}}(N), \mu_{\sigma}\left(\mathrm{WF}_{h}\left(A_{\epsilon}\right)\right)<\epsilon$, where $\mu_{\sigma}$ is the symplectic measure.

The estimate (2.8) in the second part of Lemma 2.2 shows that the contribution of $\left(1-X_{E}\right) A$ is negligible, and that the contribution of $A_{\epsilon}$ will disappear in $\epsilon \rightarrow 0$ limit.

Hence we only need to prove Theorem 1 for terms of the form $\widetilde{A}_{j, \epsilon}$. We assume now that $\rho \in B_{E} \backslash \partial B_{E}$, and that $\widetilde{A} \in \Psi_{h}^{\text {comp }}(N)$ is microlocalized in a small neighborhood $V \subset T^{*} N$ of $\rho$. Choose small $\delta>0$ and define the set

$$
U:=\left\{\varphi_{t}(\tilde{x}, \tilde{\xi}):|t|<\delta,(\tilde{x}, \tilde{\xi}) \in \Sigma_{E+\tau} \cap \pi_{E+\tau}^{-1}(V),|\tau|<\delta\right\} .
$$

If $V$ and $\delta$ are small enough, then we can write $U=U_{1} \sqcup U_{2}$, where $U_{\ell}, \ell=1,2$, are open subsets of $T^{*} M$ (one of which is a neighborhood of $\rho_{+}$and the other of $\rho_{-}$) and moreover, the maps $\kappa_{\ell}: U_{\ell} \rightarrow V \times\{|t|,|\tau|<\delta\}$,

$$
\kappa_{\ell}: \varphi_{t}(\tilde{x}, \tilde{\xi}) \longmapsto\left(\pi_{E+\tau}(\tilde{x}, \tilde{\xi}), t, \tau\right), \quad(\tilde{x}, \tilde{\xi}) \in \Sigma_{E+\tau} \cap U_{\ell},|t|,|\tau|<\delta,
$$

are diffeomorphisms. The maps $\kappa_{\ell}$ are symplectomorphisms if we consider $\{|t|,|\tau|<\delta\}$ as a subset of $T^{*} \mathbb{R}_{t}$, with $\tau$ the momentum corresponding to $t$; in fact, we provide a generating function for $\kappa_{\ell}$ in (4.6) below.

Fix a local coordinate system $x=\left(x^{\prime}, x_{n}\right)$ on $M$ such that $N=\left\{x_{n}=0\right\}$. We identify every half-density $u(x)|d x|^{1 / 2}$ on $M$ with the function $u(x)$ and every halfdensity $v(x)\left|d x^{\prime}\right|^{1 / 2}$ on $N$ with the function $v(x)$. Consider the operator

$$
\mathcal{R}: C^{\infty}(M) \rightarrow C^{\infty}\left(N \times \mathbb{R}_{t}\right), \quad \mathcal{R} u(t):=\left.\left(e^{i t(P(h)-E) / h} u\right)\right|_{N},
$$

then

$$
h D_{t} \mathcal{R}=\mathcal{R}(P(h)-E) .
$$

Take $X_{\ell} \in \Psi_{h}^{\text {comp }}(M)$ microlocalized inside $U_{\ell}$, but such that

$$
X_{\ell}=1 \text { microlocally near } \kappa_{\ell}^{-1}\left(\mathrm{WF}_{h}(\widetilde{A}) \times\{|\tau|,|t| \leq \delta / 2\}\right) .
$$

Let $\tilde{\chi}(t) \in C_{\mathrm{c}}^{\infty}(-\delta, \delta)$ be equal to 1 near $[-\delta / 2, \delta / 2]$. Then

$$
B_{\ell}:=\tilde{\chi}(t) \mathcal{R} X_{\ell}: C^{\infty}(M) \rightarrow C^{\infty}\left(N \times \mathbb{R}_{t}\right)
$$


are compactly microlocalized Fourier integral operators associated to $\kappa_{\ell}$. This follows from an oscillatory representation of $e^{i t(P(h)-E) / h}$ given in [15, §10.2]. Indeed, in coordinates $x=\left(x^{\prime}, x_{n}\right)$ and in the notation of [15, Theorem 10.4],

$$
\mathcal{B}_{\ell} u(t, \tilde{x}):=\frac{1}{(2 \pi h)^{n}} \int_{\mathbb{R}^{n}} \int_{\mathbb{R}^{n}} e^{\frac{i}{h}(\psi(t, \tilde{x}, 0, \eta)-y \cdot \eta)} b_{\ell}(t, \tilde{x}, 0, \eta ; h) u(y) d y d \eta
$$

where

$$
\begin{gathered}
\psi(0, x, \eta)=x \cdot \eta, \partial_{t} \psi\left(t, x, \partial_{x} \psi\right)=p\left(x, \partial_{x} \psi\right)-E \\
\varphi_{t}\left(x, \partial_{x} \psi(t, x, \eta)\right)=\left(\partial_{\eta} \psi(t, x, \eta), \eta\right) .
\end{gathered}
$$

The microlocalization inside $U_{\ell}$ means that $\partial_{\xi_{n}} p(\tilde{x}, 0, \xi) \neq 0$, and that implies that $\partial_{(t, \tilde{x}), \eta}^{2} \psi$ is nondegenerate. Hence, $\psi(t, \tilde{x}, 0, \eta)$ is a generating function of $\kappa_{\ell}$.

Now, let $u$ be an eigenfunction of $P(h)$ with eigenvalue $E^{\prime}=E+\lambda$, where $\lambda \in$ $[-\delta / 2, \delta / 2]$. Then $\mathrm{WF}_{h}(u) \subset p^{-1}([E-\delta / 2, E+\delta / 2])$ and thus

$$
\left.u\right|_{N}=\left.\left(X_{1}+X_{2}\right) u\right|_{N}=\left.B_{1} u\right|_{t=0}+\left.B_{2} u\right|_{t=0} \text { microlocally near } \mathrm{WF}_{h}(\widetilde{A}) .
$$

Now, by (4.4) each $w_{\ell}:=B_{\ell} u$ solves $h D_{t} w_{\ell}=\lambda w_{\ell}$ microlocally near $\mathrm{WF}_{h}(\widetilde{A})$ for $|t| \leq \delta / 2$. Therefore, $w_{\ell}(t)=e^{i t \lambda / h} w_{\ell}(0)$ microlocally near $\mathrm{WF}_{h}(\widetilde{A})$ for $|t| \leq \delta / 2$.

Take $\chi(t) \in C_{\mathrm{c}}^{\infty}(-\delta / 2, \delta / 2)$ that integrates to 1 . Then

$$
\left\langle\widetilde{A}\left(\left.w_{\ell}\right|_{t=0}\right),\left.w_{k}\right|_{t=0}\right\rangle_{L^{2}(N)}=\left\langle(\chi(t) \otimes \widetilde{A}) w_{\ell}, w_{k}\right\rangle_{L^{2}\left(N \times \mathbb{R}_{t}\right)}+\mathcal{O}\left(h^{\infty}\right) .
$$

Therefore,

$$
\begin{aligned}
\left\langle\widetilde{A}\left(\left.u\right|_{N}\right),\left(\left.u\right|_{N}\right)\right\rangle_{L^{2}(N)} & =\sum_{\ell, k=1}^{2}\left\langle\widetilde{A}\left(\left.w_{\ell}\right|_{t=0}\right),\left.w_{k}\right|_{t=0}\right\rangle_{L^{2}(N)}+\mathcal{O}\left(h^{\infty}\right) \\
& =\sum_{\ell, k=1}^{2}\left\langle(\chi(t) \otimes \widetilde{A}) w_{\ell}, w_{k}\right\rangle_{L^{2}\left(N \times \mathbb{R}_{t}\right)}+\mathcal{O}\left(h^{\infty}\right) \\
& =\sum_{\ell, k=1}^{2}\left\langle B_{k}^{*}(\chi(t) \otimes \widetilde{A}) B_{\ell} u, u\right\rangle_{L^{2}(M)}+\mathcal{O}\left(h^{\infty}\right) .
\end{aligned}
$$

We now need to analyse the operators $B_{k \ell}:=B_{k}^{*}(\chi(t) \otimes \widetilde{A}) B_{\ell}$. This is split into two cases. For $k=\ell, B_{k \ell}$ is a pseudodifferential operator and Theorem 2 can be applied with $B=B_{\ell \ell}$. Note that the operator $\chi(t) \otimes \widetilde{A}$ is not pseudodifferential, in fact its non-semiclassical wavefront set contains points $\left(t, t^{\prime}, x, x^{\prime}\right)$ with $t=t^{\prime}$ and $\left(x, x^{\prime}\right)$ in the support of the Schwartz kernel of $\widetilde{A}$. However, $B_{\ell \ell}$ is pseudodifferential since $B_{\ell}, B_{\ell}^{*}$ are compactly microlocalized and thus we can replace $\chi(t)$ by a compactly microlocalized operator in $\chi(t) \otimes A$, making the latter pseudodifferential.

We need to compute the symbol of $B_{\ell \ell}$. For that, we use the integral representation (4.5) and the stationary phase method, applicable since $\partial_{(t, \tilde{x}), \eta}^{2} \psi$ is nondegenerate. 
More precisely, the Schwartz kernel, $B_{\ell}^{*} B_{\ell}(z, y)$ is given by

$$
\frac{1}{(2 \pi h)^{2 n}} \int_{\mathbb{R}_{t, \tilde{x}}^{n} \times \mathbb{R}_{\eta}^{n} \times \mathbb{R}_{\zeta}^{n}} e^{\frac{i}{h}(\psi(t, \tilde{x}, 0, \eta)-\psi(t, \tilde{x}, 0, \zeta)+z \cdot \zeta-y \cdot \eta)} b_{\ell}(t, \tilde{x}, 0, \eta) \overline{b_{\ell}(t, \tilde{x}, 0, \zeta)} d \tilde{x} d t d \eta d \zeta .
$$

We apply the method of stationary phase in the $\tilde{x}, t, \eta$ variables. The stationary point is given by $\eta=\zeta, \varphi_{t}\left(\tilde{x}, 0, \partial_{x} \psi_{t}(t, \tilde{x}, 0, \eta)\right)=(y, \eta)$, and the value of the phase at the stationary point is $(z-y) \cdot \zeta$. The signature of the Hessian is 0 and, by (4.6) and [15, Theorem 10.6], the leading part of the symbol in the region of interest is given by

$$
\left|\frac{\operatorname{det} \partial_{x} \partial_{\zeta} \psi(t, \tilde{x}, 0, \zeta)}{\operatorname{det} \partial_{(t, \tilde{x})} \partial_{\zeta} \psi(t, \tilde{x}, 0, \zeta)}\right|=\frac{1}{\left|\partial_{\xi_{n}} p\left(\tilde{x}, 0, \partial_{x} \psi(t, \tilde{x}, 0, \zeta)\right)\right|},
$$

where $t=t(y, \zeta), \tilde{x}=\tilde{x}(y, \zeta)$, are the critical points.

Recalling (4.2), it follows that

$$
\sigma\left(B_{\ell}^{*} B_{\ell}\right) \circ \kappa_{\ell}^{-1}=\left|\partial_{\xi_{n}} p \circ \kappa_{\ell}^{-1} \circ \pi_{0}\right|^{-1} \text { near } \mathrm{WF}_{h}(\widetilde{A}) \times\{|\tau|,|t| \leq \delta / 2\},
$$

where $\pi_{0}: T^{*}\left(N \times \mathbb{R}_{t}\right) \rightarrow T^{*}\left(N \times \mathbb{R}_{t}\right)$ maps $(\tilde{x}, \tilde{\xi}, t, \tau)$ to $(\tilde{x}, \tilde{\xi}, 0, \tau)$. From here and by Egorov's Theorem applied to $\chi(t) \otimes \widetilde{A}$, we get $\sigma\left(B_{\ell \ell}\right) \circ \kappa_{\ell}^{-1}=\left|\partial_{\xi_{n}} p \circ \kappa_{\ell}^{-1} \circ \pi_{0}\right|^{-1} \sigma(\widetilde{A}) \chi(t)$ near $\{|\tau| \leq \delta / 2\}$. Then

$$
\begin{gathered}
\int_{\left\{p=E_{j}\right\}} \sigma\left(B_{\ell \ell}\right) d \mu_{E_{j}}=\frac{1}{\mu_{E_{j}}\left(p^{-1}\left(E_{j}\right)\right)} \int_{\left\{\tau=E_{j}-E\right\}} \sigma\left(B_{\ell \ell}\right) \circ \kappa_{\ell}^{-1} d \tilde{x} d \tilde{\xi} d t \\
=\frac{1}{\mu_{E_{j}}\left(p^{-1}\left(E_{j}\right)\right)} \int_{\Sigma_{E_{j}} \cap U_{\ell}}\left|\partial_{\xi_{n}} p\right|^{-1} \pi_{E_{j}}^{*} \sigma(\widetilde{A}) d x^{\prime} d \xi^{\prime}
\end{gathered}
$$

where we parametrized $\Sigma_{E_{j}}$ by $\left(x^{\prime}, \xi^{\prime}\right) \in B_{E_{j}}$.

Now, we consider the case $k \neq \ell$. Then $B_{k \ell}$ is a Fourier integral operator with the canonical transformation $\kappa_{k \ell}:=\kappa_{k}^{-1} \circ \kappa_{\ell}$. We want to apply the decorrelation result given in Lemma 3.1.

Using the definition (4.2) of $\kappa_{\ell}$, we see that the canonical transformation $\kappa=\kappa_{k \ell}$ can be described as follows:

$$
\kappa\left(\varphi_{s}(\tilde{x}, \tilde{\xi})\right)=\varphi_{s}\left(\gamma_{E^{\prime}}(\tilde{x}, \tilde{\xi})\right),|s|<\delta,(\tilde{x}, \tilde{\xi}) \in \Sigma_{E^{\prime}} \cap U_{\ell}
$$

To apply Lemma 3.1 we need to verify the following: there exists $t_{0}>0$ such that the set

$$
\mathcal{E}:=\left\{\rho \in U_{\ell} \cap \varphi_{-t}\left(U_{\ell}\right): \exists t,|t| \geq t_{0}, \varphi_{t}(\kappa(\rho))=\kappa\left(\varphi_{t}(\rho)\right)\right\} \subset T^{*} M,
$$

has $\mu_{\sigma}$-measure zero. To see this, suppose that $\rho \in \mathcal{E}, t$ is the corresponding time, and $s, s^{\prime} \in(-\delta, \delta)$ are such that $\rho=\varphi_{s}(\tilde{x}, \tilde{\xi}), \varphi_{t}(\rho)=\varphi_{s^{\prime}}\left(\tilde{x}^{\prime}, \tilde{\xi}^{\prime}\right),(\tilde{x}, \tilde{\xi}),\left(\tilde{x}^{\prime}, \tilde{\xi}^{\prime}\right) \in \Sigma_{E^{\prime}} \cap U_{\ell}$. Then $\left(\tilde{x}^{\prime}, \tilde{\xi}^{\prime}\right)=\varphi_{t+s-s^{\prime}}(\tilde{x}, \tilde{\xi})$ and the condition $\varphi_{t}(\kappa(\rho))=\kappa\left(\varphi_{t}(\rho)\right)$ can be rewritten as

$$
(\tilde{x}, \tilde{\xi}) \in \Sigma_{E^{\prime}}, \varphi_{t+s-s^{\prime}}(\tilde{x}, \tilde{\xi}) \in \Sigma_{E^{\prime}}, \varphi_{t+s-s^{\prime}}\left(\gamma_{E^{\prime}}(\tilde{x}, \tilde{\xi})\right)=\gamma_{E^{\prime}}\left(\varphi_{t+s-s^{\prime}}(\tilde{x}, \tilde{\xi})\right) .
$$


Put $t_{0}>2 \delta$, then $t+s-s^{\prime} \neq 0$. It now follows from (1.7) that the set $\mathcal{E}$ from (4.9) has measure zero; by Lemma 3.1, the contributions of $B_{k \ell}, k \neq \ell$ to the sum (1.9) go to 0 as $h \rightarrow 0$.

Going back to (4.7), (A.4) and (4.8) this means for $\widetilde{A}$ satisfying our localization assumptions

$$
\left.h^{n} \sum_{E_{j} \in[a, b]}\left|\left\langle\widetilde{A}\left(\left.u_{j}\right|_{N}\right),\left(\left.u_{j}\right|_{N}\right)\right\rangle_{L^{2}(N)}-\frac{1}{V_{j}} \sum_{\ell=1,2} \int_{\Sigma_{E_{j}} \cap U_{\ell}}\right| \partial_{\xi_{n}} p\right|^{-1} \pi_{E_{j}}^{*} \sigma(\widetilde{A}) d x^{\prime} d \xi^{\prime} \mid=o(1),
$$

where $V_{j}:=\mu_{E_{j}}\left(p^{-1}\left(E_{j}\right)\right)$. Assume that the function $f$ used to define restrictions of half-densities in (1.8) is equal to $x_{n}$, so that $u(x)|d x|^{1 / 2}$ restricts to $u(\tilde{x}, 0)\left|d x^{\prime}\right|^{1 / 2}$. Using the canonical transformation $\kappa_{\ell}$, we get the symplectic coordinates $\left(x^{\prime}, t, \xi^{\prime}, \tau\right)$ on $U_{\ell}$, in which the measure $d \nu_{E_{j}}$ from (1.10) is equal to $V_{j}^{-1}\left|\partial_{\xi_{n}} p\right|^{-1} d x^{\prime} d \xi^{\prime}$. We have thus proved (1.9) with $f=x_{n}$; the case of general $f$ follows by taking the operator $\left|\partial_{x_{n}} f\right|^{-1 / 2} A\left|\partial_{x_{n}} f\right|^{-1 / 2}$ in place of $A$. This completes the proof of Theorem 1.

\section{Appendix A. Semiclassical quantum ergodicity With Boundaries}

Let $(M, g)$ be a smooth Riemannian manifold with a piecewise smooth boundary, $\partial M$. That means that $M \subset \widetilde{M}$ where $\widetilde{M}$ is manifold without boundary to which $g$ extends smoothly, and $\partial M=\bigcup_{j=1}^{J} N_{j}$ where $N_{j}$ are smooth embedded hypersurfaces in $\widetilde{M}$. Denote by $\partial^{\circ} M \subset \partial M$ the open set of all points at which the boundary is smooth, namely points contained in exactly one of the hypersurfaces $N_{j}$; the complement $\partial M \backslash$ $\partial^{\circ} M$ has measure zero (with respect to the surface measure on $\partial M$ ).

We consider an operator $P(h)$ given by (1.1) with Dirichlet boundary conditions. (One can take instead any self-adjoint boundary conditions, as long as the Weyl law (A.3) is known to hold.) Let $p(x, \xi)$ be its principal symbol; we can extend it smoothly to $T^{*} \widetilde{M}$. We make the following assumption similar to (1.5), for $E \in[a, b]$ :

$$
x \in \partial^{\circ} M, V(x)=E \Longrightarrow d V(x) \notin N_{x}^{*} \partial M .
$$

Then $p^{-1}(E)$ and $T_{\partial^{\circ} M}^{*} M$ intersect transversally. We write

$$
p^{-1}(E) \cap T_{\partial^{\circ} M}^{*} M=\Omega_{E}^{+} \sqcup \Omega_{E}^{-} \sqcup \Omega_{E}^{0},
$$

where $(x, \xi)$ lies in $\Omega_{E}^{+}$if the vector $H_{p} x \in T \widetilde{M}$ is pointing outside of $M$, in $\Omega_{E}^{-}$if this vector is pointing inside $M$, and in $\Omega_{E}^{0}$ if $H_{p} x$ is tangent to the boundary of $M$. The covectors in $\Omega_{E}^{0}$ are called glancing, and under the assumption (A.1) this set has measure zero inside $p^{-1}(E) \cap T_{\partial}^{*} M M$.

For $(x, \xi) \in p^{-1}(E)$, we define its broken Hamiltonian flow line $\varphi_{t}(x, \xi)$ as follows. Assuming without loss of generality that $t>0$, we consider the Hamiltonian flow line $\exp \left(t H_{p}\right)(x, \xi)$, defined smoothly on $T^{*} \widetilde{M}$, and let $t_{0}$ be the first nonnegative time when 
$\exp \left(t H_{p}\right)(x, \xi)$ hits the boundary. If this happens at a non-smooth point of the boundary (i.e. on $\left.\partial M \backslash \partial^{\circ} M\right)$, or if $\exp \left(t_{0} H_{p}\right)(x, \xi) \in \Omega_{E}^{0}$, then the flow cannot be extended past $t=t_{0}$. Otherwise, $\exp \left(t_{0} H_{p}\right)(x, \xi) \in \Omega_{E}^{+}$and there exists unique $\left(x_{0}, \xi_{0}\right) \in \Omega_{E}^{-}$ such that the natural projections of $\exp \left(t_{0} H_{p}\right)(x, \xi)$ and $\left(x_{0}, \xi_{0}\right)$ onto $T^{*} \partial M$ are the same. We then define $\varphi_{t}$ inductively, by putting $\varphi_{t}(x, \xi)=\exp \left(t H_{p}\right)(x, \xi)$ for $0<t<t_{0}$ and $\varphi_{t}(x, \xi)=\varphi_{t-t_{0}}\left(x_{0}, \xi_{0}\right)$ for $t>t_{0}$. For any $T>0$, denote by

$$
\mathcal{B}_{T} \subset T^{*} M \cap p^{-1}([a, b])
$$

the closed set of all $(x, \xi)$ such one cannot define the flow $\varphi_{t}(x, \xi)$ on the interval $[-T, T]$ using the above procedure. As shown in [14, Lemma 1], for any $T$ the set $\mathcal{B}_{T} \cap p^{-1}(E)$ has measure zero in $p^{-1}(E)$, and for $|t| \leq T, \varphi_{t}$ is a volume preserving flow on $p^{-1}(E) \backslash \mathcal{B}_{T}$. See also [8, p. 310-311] for a symplectically invariant description of the broken Hamiltonian flow. Since the flow $\varphi_{t}$ is well-defined almost everywhere, the standard ergodic theory applies to it.

We will use the following parametrix construction for the Schrödinger propagator away from the set $\mathcal{B}_{T}$. The following lemma is a rephrasing of results of Christianson [2, $\S 3.3]$ :

Lemma A.1. Fix $T>0$. Assume that $A \in \Psi_{h}^{\mathrm{comp}}\left(M^{\circ}\right)$ is supported away from the boundary of $M$ and $\mathrm{WF}_{h}(A) \subset p^{-1}([a, b]) \backslash \mathcal{B}_{T}$. Then for each $\chi \in C_{c}^{\infty}\left(M^{\circ}\right)$ and for each $t \in[-T, T]$, the operator $\chi e^{-i t P / h} A$ is a Fourier integral operator supported away from $\partial M$ and associated to the restriction of $\varphi_{t}$ to a neighborhood of $\mathrm{WF}_{h}(A) \cap$ $\varphi_{t}^{-1}(\operatorname{supp} \chi)$, plus an $\mathcal{O}\left(h^{\infty}\right)_{L^{2}(M) \rightarrow L^{2}(M)}$ remainder. The following version of Egorov's Theorem holds:

$$
\chi e^{i t P / h} A e^{-i t P / h}=A_{t, \chi}+\mathcal{O}\left(h^{\infty}\right)_{L^{2}(M) \rightarrow L^{2}(M)},
$$

where $A_{t, \chi} \in \Psi_{h}^{\mathrm{comp}}\left(M^{\circ}\right)$ is supported away from $\partial M$ and $\sigma\left(A_{t, \chi}\right)=\chi\left(a \circ \varphi_{t}\right)$.

The following basic Weyl law can be proved for the Dirichlet realization of $P(h)$ using the standard Dirichlet-Neumann bracketing method (see [9, Chapter 15]):

$$
(2 \pi h)^{n} \#\left\{j: E_{j} \in[a, b]\right\}=\mu_{\sigma}\left(T^{*} M \cap p^{-1}([a, b])\right)+o(1) \text { as } h \rightarrow 0 .
$$

It follows that eigenfunctions cannot on average concentrate near the boundary:

Lemma A.2. Assume that $\chi \in C_{c}^{\infty}\left(M^{\circ}\right)$ satisfies $0 \leq \chi \leq 1$. Then for $a^{\prime}<a<b<b^{\prime}$,

$$
(2 \pi h)^{n} \sum_{E_{j} \in[a, b]} \int_{M}(1-\chi)\left|u_{j}\right|^{2} d \mathrm{Vol} \leq \int_{T^{*} M \cap p^{-1}\left(\left[a^{\prime}, b^{\prime}\right]\right)} 1-\chi d \mu_{\sigma}+o(1) \text { as } h \rightarrow 0 .
$$

Proof. Take $f \in C_{c}^{\infty}\left(a^{\prime}, b^{\prime}\right)$ such that $0 \leq f \leq 1$ and $f=1$ near $[a, b]$. Since $f$ and $1-\chi$ are nonnegative, it suffices to show that

$$
(2 \pi h)^{n} \sum_{j} \int_{M}(1-\chi) f\left(E_{j}\right)\left|u_{j}\right|^{2} d \mathrm{Vol}=\int_{T^{*} M}(1-\chi) f(p) d \mu_{\sigma}+o(1) \text { as } h \rightarrow 0
$$


This holds since the asymptotics for 1 in place of $1-\chi$ follows from (A.3), while the asymptotics for $\chi$ follows from (2.6).

We can now prove quantum ergodicity for manifolds with boundary:

Theorem 2. Suppose that $(M, g)$ is a compact manifold with a piecewise smooth boundary and that $u_{j}=u_{j}(h)$ are normalized eigenfuctions of the Dirichlet realization of $P(h)$. If (1.3) and (A.1) hold, then for any $B \in \Psi_{h}^{0}\left(M^{\circ}\right)$ compactly supported away from $\partial M$,

$$
h^{n} \sum_{E_{j} \in[a, b]}\left|\left\langle B u_{j}, u_{j}\right\rangle_{L^{2}(M)}-f_{p^{-1}\left(E_{j}\right)} \sigma(B) d \mu_{E_{j}}\right| \longrightarrow 0, \quad h \rightarrow 0 .
$$

Proof. Take $a^{\prime}, b^{\prime}$ such that $a^{\prime}<a<b<b^{\prime}$ and (1.3) and (A.1) hold for $E \in\left[a^{\prime}, b^{\prime}\right]$. (If the flow is no longer ergodic on $p^{-1}(E)$ when $E \notin[a, b]$, we would need to consider $a^{\prime}, b^{\prime}$ close to $a, b$, for example $a^{\prime}=a-1 / T$ and $b^{\prime}=b+1 / T$ and crudely estimate the contribution of $\left[a^{\prime}, b^{\prime}\right] \backslash[a, b]$ by the Weyl law.) Take large $T>0$ and choose a cutoff function $\chi_{T} \in C_{c}^{\infty}\left(M^{\circ}\right)$ such that $0 \leq \chi_{T} \leq 1$ and

$$
\int_{T^{*} M \cap p^{-1}\left(\left[a^{\prime}-1, b^{\prime}+1\right]\right)} 1-\chi_{T} d \mu_{\sigma} \leq T^{-1} .
$$

Let the function $\psi \in C_{c}^{\infty}\left(a^{\prime}-1, b^{\prime}+1\right)$ satisfy

$$
\psi(E) \int_{p^{-1}(E)} \chi_{T} d \mu_{E}=\int_{p^{-1}(E)} \sigma(B) d \mu_{E}, E \in\left[a^{\prime}, b^{\prime}\right] .
$$

By Lemma A.2, it is enough to show that for $T$ arbitrarily large but fixed, (A.4) holds for the operator $B-\psi(P(h)) \chi_{T}$, whose symbol integrates to zero on $p^{-1}(E)$ for $E \in\left[a^{\prime}, b^{\prime}\right]$; therefore, without loss of generality we assume that

$$
\int_{p^{-1}(E)} \sigma(B) d \mu_{E}=0, E \in\left[a^{\prime}, b^{\prime}\right]
$$

By the elliptic estimate (see for instance [5, Proposition 3.2]), we may assume that $\mathrm{WF}_{h}(B) \subset p^{-1}\left(\left(a^{\prime}, b^{\prime}\right)\right)$ and in particular $B \in \Psi_{h}^{\mathrm{comp}}$. The set $\mathcal{B}_{T}$ defined in (A.2) is closed and has measure zero; therefore, we can write $B=B_{T}^{\prime}+B_{T}^{\prime \prime}$, where $\mathrm{WF}_{h}\left(B_{T}^{\prime}\right) \cap$ $\mathcal{B}_{T}=\emptyset$ and $\left\|\sigma\left(B_{T}^{\prime \prime}\right)\right\|_{L^{2}\left(p^{-1}\left[a^{\prime}, b^{\prime}\right]\right)} \leq T^{-1}$. By (2.7), the contribution of $B_{T}^{\prime \prime}$ to (A.4) goes to zero in the limit $\lim _{T \rightarrow \infty} \lim \sup _{h \rightarrow 0}$; therefore, we can replace $B$ by $B_{T}^{\prime}$ in (A.4). Define the quantum averaged operator

$$
\left\langle B_{T}^{\prime}\right\rangle_{T}:=\frac{1}{T} \int_{0}^{T} e^{i t P / h} B_{T}^{\prime} e^{-i t P / h} d t
$$


Then by Lemma A.1, $\left\langle B_{T}^{\prime}\right\rangle_{T} \chi_{T}$ is, up to an $\mathcal{O}\left(h^{\infty}\right)_{L^{2} \rightarrow L^{2}}$ remainder, a pseudodifferential operator in $\Psi_{h}^{\text {comp }}$ compactly supported inside $M^{\circ}$ and with principal symbol

$$
\sigma\left(\left\langle B_{T}^{\prime}\right\rangle_{T} \chi_{T}\right)=\chi_{T}\left\langle\sigma\left(B_{T}^{\prime}\right)\right\rangle_{T}=\frac{\chi_{T}}{T} \int_{0}^{T} \sigma\left(B_{T}^{\prime}\right) \circ \varphi_{t} d t
$$

Since each $u_{j}$ is an eigenvalue of $P(h)$, we can write the left-hand side of (A.4) as

$$
h^{n} \sum_{E_{j} \in[a, b]}\left|\left\langle\left\langle B_{T}^{\prime}\right\rangle_{T} u_{j}, u_{j}\right\rangle\right|
$$

using the Weyl law (A.3) and Cauchy-Schwarz, we see that it remains to prove that

$$
\lim _{T \rightarrow \infty} \limsup _{h \rightarrow 0} h^{n} \sum_{E_{j} \in[a, b]}\left\|\left\langle B_{T}^{\prime}\right\rangle_{T} u_{j}\right\|_{L^{2}}^{2}=0 .
$$

We can replace $\left\langle B_{T}^{\prime}\right\rangle_{T}$ here by $\left\langle B_{T}^{\prime}\right\rangle_{T} \chi_{T}$, as

$$
\limsup _{h \rightarrow 0}(2 \pi h)^{n} \sum_{E_{j} \in[a, b]}\left\|\left(1-\chi_{T}\right) u_{j}\right\|_{L^{2}}^{2} \leq T^{-1}
$$

by Lemma A.2. By (2.7), it remains to show that

$$
\lim _{T \rightarrow \infty}\left\|\sigma\left(\left\langle B_{T}^{\prime}\right\rangle_{T} \chi_{T}\right)\right\|_{L^{2}\left(p^{-1}\left(\left[a^{\prime}, b^{\prime}\right]\right)\right)} \leq \lim _{T \rightarrow \infty}\left\|\left\langle\sigma\left(B_{T}^{\prime}\right)\right\rangle_{T}\right\|_{L^{2}\left(p^{-1}\left(\left[a^{\prime}, b^{\prime}\right]\right)\right)}=0 .
$$

For this, we write for each $E \in\left[a^{\prime}, b^{\prime}\right]$,

$$
\left\|\left\langle\sigma\left(B_{T}^{\prime}\right)\right\rangle_{T}\right\|_{L^{2}\left(p^{-1}(E)\right)} \leq\left\|\langle\sigma(B)\rangle_{T}\right\|_{L^{2}\left(p^{-1}(E)\right)}+\left\|\left\langle\sigma\left(B_{T}^{\prime \prime}\right)\right\rangle_{T}\right\|_{L^{2}\left(p^{-1}(E)\right)} .
$$

The first term on the right-hand side converges to 0 when $T \rightarrow \infty$ by (A.5) and the von Neumann ergodic theorem (see for example [15, Theorem 15.1]), while the second term is bounded by $\left\|\sigma\left(B_{T}^{\prime \prime}\right)\right\|_{L^{2}\left(p^{-1}(E)\right)}$.

\section{APPENDIX B. FROM SEMICLASSICAL TO HIGH ENERGY ASYMPTOTICS}

In this appendix we specialize to $P(h)=-h^{2} \Delta_{g}$ and show how Theorem 1 implies the results of [13].

Suppose that $(M, g)$ is a compact Riemannian manifold with a piecewise smooth boundary in the sense of Appendix A, and with an ergodic broken geodesic flow $\varphi_{t}$ : $S^{*} M \rightarrow S^{*} M$. Suppose that $N \subset M$ is an open smooth hypersurface whose closure is disjoint from the boundary. The energy surface $p^{-1}(1)=S^{*} M$ is the cosphere bundle of $M$ and $\Sigma_{1}=S_{N}^{*} M$ is the restriction of $S^{*} M$ to $N ; B_{1}=\pi_{1}\left(\Sigma_{1}\right)=B^{*} N$ is the coball bundle of $N$ and $\gamma_{1}: \Sigma_{1} \rightarrow \Sigma_{1}$ is the reflection across the orthogonal complement of the conormal bundle $N^{*} N \subset T_{N}^{*} M$.

The dynamical assumption (1.7) becomes

The set of $\rho \in S_{N}^{*} M$ satisfying $\varphi_{t}(\rho) \in S_{N}^{*} M$

and $\varphi_{t}\left(\gamma_{1}(\rho)\right)=\gamma_{1}\left(\varphi_{t}(\rho)\right)$ for some $t \neq 0$, has measure 0 . 
Let $\left\{u_{j}\right\}_{j=0}^{\infty}$ be the complete set of eigenfunctions of the Laplacian on $(M, g)$ :

$$
-\Delta_{g} u_{j}=\lambda_{j}^{2} u_{j}, \quad\left\|u_{j}\right\|_{L^{2}}=1, \quad 0=\lambda_{0}<\lambda_{1} \leq \lambda_{2} \leq \cdots .
$$

The statement of the theorem uses the standard concept of a (nonsemiclassical) pseudodifferential operator on a manifold - see $[8, \S 18.2]$.

Theorem 3. Let $N$ be a smooth open hypersurface satisfying (B.1) with closure disjoint from the boundary. Suppose that $A \in \Psi_{\mathrm{phg}}^{0}(N)$ is a classical pseudodifferential operator on $N$, compactly supported inside $N$. Put $v_{j}:=\left.u_{j}\right|_{N}$. Then

$$
\frac{1}{\lambda^{n}} \sum_{\lambda_{j} \leq \lambda}\left|\left\langle A v_{j}, v_{j}\right\rangle_{L^{2}\left(N, d \operatorname{vol}_{g \mid}\right)}-\int_{S_{N}^{*} M} \pi_{1}^{*} \sigma(A) d \nu_{1}\right| \longrightarrow 0, \quad \lambda \rightarrow \infty,
$$

where $\sigma(A)$ is the principal symbol of $A$ (a homogeneous function of degree 0 on $T^{*} N \backslash$ $\{0\})$, and the measure $\nu_{1}$ is defined in (1.11).

Remark. Theorem 1 allows more general restrictions $\left.a u_{j}\right|_{N}+\left.b \lambda_{j}^{-1} \partial_{\nu} u_{j}\right|_{N}$, for $a, b \in$ $C^{\infty}(N)$. We note also that the nonsemiclassical formulation of quantum ergodicity only implies the angular equidistribution of $v_{j}$ in $T_{x}^{*} N$. That is natural for the standard quantum ergodicity since $u_{j}$ concentrate on $S^{*} M$ but not in this case as $v_{j}$ 's can be microsupported anywhere in $B^{*} N$. That is remedied in the semiclassical Theorem 1.

Proof. To show how Theorem 3 follows from Theorem 1 we put $V \equiv 0$ and identify $L^{2}\left(M, \Omega_{M}^{1 / 2}\right)$ with $L^{2}\left(M, d \operatorname{vol}_{g}\right)$ by writing half-densities as $u(x)\left|d \operatorname{vol}_{g}\right|^{1 / 2}$, where $u \in$ $L^{2}\left(M, d \operatorname{vol}_{g}\right)$.

Let $x=\left(x^{\prime}, x_{n}\right)$ be normal geodesic coordinates near $(0,0) \in N$, in which $N=$ $\left\{x_{n}=0\right\}, p\left(x, \xi^{\prime}, \xi_{n}\right)=\xi_{n}^{2}+r\left(x, \xi^{\prime}\right)$, and $r\left(x^{\prime}, 0, \xi^{\prime}\right)$ is the dual of the restriction metric $\left.g\right|_{N}$. Suppose that $f$ satisfies $\left.f\right|_{N}=0,|d f(x)|_{g}=1$. In the chosen coordinates the last condition means that $\partial_{x_{n}} f=1, f=0$, on $N$. Hence the restriction of half-densities (1.8) obtained using this choice of $f$ shows that, we obtain an identification with the restriction of functions $\left.u\right|_{N} \in L^{2}\left(N, d \operatorname{vol}_{\left.g\right|_{N}}\right)$.

We now write out locally the measure $\nu_{1}$ from (1.10). In our coordinates, $S^{*} M$ can be parametrized by $\left(x^{\prime}, x_{n}, \xi^{\prime}\right), r\left(x^{\prime}, \xi^{\prime}\right) \leq 1, \xi_{n}= \pm\left(1-r\left(x, \xi^{\prime}\right)\right)^{1 / 2}$ (the parametrization degenerates at $\left.r\left(x, \xi^{\prime}\right)=1\right)$. The Liouville measure is obtained by requiring $d \mu_{1} \wedge d p=$ $d x d \xi$, and

$$
d \mu_{1}=\frac{1}{2\left|\xi_{n}\right|} d x d \xi^{\prime}=\frac{1}{2\left(1-r\left(x, \xi^{\prime}\right)\right)^{1 / 2}} d x d \xi^{\prime} .
$$

In the notation of (1.11) this gives

$$
d \nu_{1}=\frac{1}{\mu_{1}\left(S^{*} M\right)} \frac{1}{2 \sqrt{1-r\left(x^{\prime}, 0, \xi^{\prime}\right)}} d x^{\prime} d \xi^{\prime}, \quad B_{1}=\left\{\left(x^{\prime}, \xi^{\prime}\right): r\left(x^{\prime}, 0, \xi^{\prime}\right) \leq 1\right\},
$$

where we parametrized $S_{N}^{*} M=\left\{\left(x^{\prime}, 0, \xi\right): \xi_{n}^{2}+r\left(x^{\prime}, 0, \xi^{\prime}\right)=1\right\}$ by $\left(x^{\prime}, \xi^{\prime}\right) \in B_{1}$. 
To pass from the semiclassical result to the special case of the high energy result we put $E_{j}=h^{2} \lambda_{j}^{2}, h=1 / \lambda$. The difficulty lies in controlling low frequency contributions and estimates (2.8) and (2.9) are crucial for that.

Let $\widehat{A}$ be a classical pseudodifferential operator of order 0 on $N$, with a compactly supported Schwartz kernel in $N$. (Henceforth operators with hats denote polyhomogeneous operators, while operators without hats denote semiclassical operators.) Its principal symbol $\sigma(\widehat{A})$ is a homogeneous function of degree 0 on $T^{*} N$. We define $A_{\epsilon} \in \Psi_{h}^{0}(N)$ by putting

$$
A_{\epsilon}:=\mathrm{Op}_{h}\left(\sigma(\widehat{A})\left(1-\chi\left(\left|\xi^{\prime}\right|_{\left.g\right|_{N}} / \epsilon\right)\right)\right), \quad \chi \in C_{\mathrm{c}}^{\infty}(\mathbb{R}), \quad \chi(t)=1, \quad|t| \leq 1 .
$$

Theorem 1 shows that for $0<a<b$, and $v_{j}=\left.u_{j}\right|_{N}$,

$$
h^{n} \sum_{h \lambda_{j} \in[a, b]}\left|\left\langle A_{\epsilon} v_{j}, v_{j}\right\rangle_{L^{2}\left(N, d \operatorname{vol}_{\left.g\right|_{N}}\right)}-\int_{\Sigma_{E_{j}}} \pi_{E_{j}}^{*} \sigma\left(A_{\epsilon}\right) d \nu_{E_{j}}\right| \longrightarrow 0, \quad h=1 / \lambda \rightarrow 0 .
$$

We also have

$$
\int_{\Sigma_{E_{j}}} \pi_{E_{j}}^{*} \sigma\left(A_{\epsilon}\right) d \nu_{E_{j}}=\int_{S_{N}^{*} M} \pi_{1}^{*} \sigma(\widehat{A}) d \nu_{1}+\mathcal{O}(\epsilon)
$$

and hence the result will follow once we show that

$$
h^{n} \sum_{h \lambda_{j} \in[a, b]}\left|\left\langle\left(\widehat{A}-A_{\epsilon}\right) v_{j}, v_{j}\right\rangle_{L^{2}\left(N, d \operatorname{vol}_{\left.g\right|_{N}}\right)}\right|=\mathcal{O}(\epsilon)+\mathcal{O}_{\epsilon}(h) .
$$

Indeed, (B.3) and (B.4) together give, for $[a, b]=[1,2]$,

$$
h^{n} \sum_{h \lambda_{j} \in[1,2]}\left|\left\langle\widehat{A} v_{j}, v_{j}\right\rangle_{L^{2}\left(N, d \operatorname{vol}_{\left.g\right|_{N}}\right)}-\int_{S_{N}^{*} M} \pi_{1}^{*} \sigma(\widehat{A}) d \nu_{1}\right| \rightarrow 0 \text { as } h \rightarrow 0 .
$$

Summing (B.5) for $h=2^{k} \lambda^{-1}, 1 \leq k \leq \log _{2} \lambda$, we get (B.2).

We now prove (B.4). Using (2.9), it will follow from

$$
\limsup _{\epsilon \rightarrow 0} \limsup _{h \rightarrow 0} \sum_{h \lambda_{j} \in[a, b]}\left\|\left(\widehat{A}-A_{\epsilon}\right) v_{j}(h)\right\|^{2}=0 .
$$

For this, we first claim that for any vector field $X$ on $N$,

$$
\left\|\left(\widehat{A}-A_{\epsilon}\right) h X\right\|_{L^{2} \rightarrow L^{2}} \leq C \epsilon+\mathcal{O}_{\epsilon}(h) .
$$

Indeed, the left-hand side of (B.7) is $\mathcal{O}(h)$ if we put an operator in the class $\Psi_{\text {phg }}^{-1}$ or $h \Psi_{h}^{-1}$ in place of $\widehat{A}-A_{\epsilon}$, which means that we can reduce to local coordinates, in which we can assume $X=\partial_{y_{1}}$ and the full symbol of $\left(\widehat{A}-A_{\epsilon}\right) h X$ in the non-semiclassical left quantization becomes, up to $\Psi_{\mathrm{phg}}^{-1}+h \Psi_{h}^{-1}$ terms,

$$
r(y, \eta ; h):=h \eta_{1} a^{0}(y, \eta /|\eta|)\left((1-\chi(|\eta|))-\left(1-\chi\left(h|\eta|_{\left.g\right|_{N}} / \epsilon\right)\right)\right) .
$$


However, $\partial_{y}^{\alpha} \partial_{\eta}^{\beta} r(y, \eta ; h)=\mathcal{O}(\epsilon+h)\langle\eta\rangle^{-|\beta|}$ (here the first cutoff gives the $\mathcal{O}(h)$ term, while the second cutoff gives the $\mathcal{O}(\epsilon)$ term); therefore, by the $L^{2}$ boundedness of classical pseudodifferential operators, we get (B.7).

Now, let $B_{0} \in \Psi_{h}^{\text {comp }}(N)$ be a semiclassical pseudodifferential operator equal to the identity microlocally near the zero section of $T^{*} N$, but supported inside an $\epsilon^{1 / 2}$ sized neighborhood of the zero section. Then we can write

$$
1-B_{0}=\sum_{k}\left(h X_{k}\right) B_{0}^{k}+\mathcal{O}_{\epsilon}(h)_{L^{2} \rightarrow L^{2}}
$$

for some vector fields $X_{k}$ (independent of $\epsilon$ ) and some $B_{0}^{k} \in \Psi_{h}^{\text {comp }}(N)$ (with $L^{2} \rightarrow L^{2}$ norm $\left.\mathcal{O}\left(\epsilon^{-1 / 2}\right)\right)$; by $(\mathrm{B} .7)$, we have

$$
\left\|\left(\widehat{A}-A_{\epsilon}\right)\left(1-B_{0}\right)\right\|_{L^{2} \rightarrow L^{2}} \leq C \epsilon^{\frac{1}{2}}+\mathcal{O}_{\epsilon}(h)
$$

and thus by (2.9), the estimate (B.6) holds for $\left(\widehat{A}-A_{\epsilon}\right)\left(1-B_{0}\right)$. Same estimate holds for $\left(\widehat{A}-A_{\epsilon}\right) B_{0}$, by recalling that $\left\|\widehat{A}-A_{\epsilon}\right\|_{L^{2} \rightarrow L^{2}}=\mathcal{O}(1)$ and using (2.8) together with the bound $\left\|\sigma\left(B_{0}\right)\right\|_{L^{2}} \leq \epsilon^{(n-1) / 4}$. This finishes the proof of (B.6) and thus of Theorem 3.

\section{REFERENCES}

[1] N. Burq, P. Gérard and N. Tzvetkov, Restrictions of the Laplace-Beltrami eigenfunctions to submanifolds, Duke Math. J. 138(2007), no. 3, 445-486.

[2] H. Christianson, Quantum monodromy and non-concentration near a closed semi-hyperbolic orbit, Trans. Amer. Math. Soc. 363(2011), no. 7, 3373-3438.

[3] H. Christianson, J. Toth, and S. Zelditch, Quantum ergodic restriction for Cauchy data: interior QUE and restricted QUE, preprint, arXiv:1205.0286.

[4] S. Dyatlov, Asymptotic distribution of quasi-normal modes for Kerr-de Sitter black holes, to appear in Ann. Henri Poincaré, arXiv:1101.1260.

[5] S. Dyatlov and C. Guillarmou, Microlocal limits of plane waves and Eisenstein functions, preprint, arXiv:1204.1305

[6] P. Gérard and E. Leichtnam, Ergodic properties of eigenfunctions for the Dirichlet problem, Duke Math. J. 71(1993), no. 2, 559-607.

[7] B. Helffer, A. Martinez, and D. Robert, Ergodicité en limite semi-classique, Comm. Math. Phys. 109(1987), no. 2, 313-326.

[8] L. Hörmander, The Analysis of Linear Partial Differential Operators III. Pseudo-differential Operators, Springer, 1985.

[9] M. Reed and B. Simon, Methods of Modern Mathematical Physics IV. Analysis of Operators, New York, Academic Press, 1978.

[10] J. Sjöstrand and M. Zworski, Quantum monodromy and semiclassical trace formulae, J. Math. Pure Appl. 81(2002), no. 1, 1-33.

[11] M. Tacy, Semiclassical $L^{p}$ estimates of quasimodes on submanifolds, Comm. P.D.E. 35(2010), no. $8,1538-1562$.

[12] J.A. Toth and S. Zelditch, Quantum ergodic restriction theorems. I: interior hypersurfaces in domains with ergodic billiards, Ann. Henri Poincaré 13(2012), 599-670. 
[13] J.A. Toth and S. Zelditch, Quantum ergodic restriction theorems, II: manifolds without boundary, preprint, arXiv:1104.4531.

[14] S. Zelditch and M. Zworski, Ergodicity of eigenfunctions for ergodic billiards, Comm. Math. Phys. 175(1996), no. 3, 673-682.

[15] M. Zworski, Semiclassical analysis, Graduate Studies in Mathematics 138, AMS, 2012.

E-mail address: dyatlov@math.berkeley.edu

E-mail address: zworski@math.berkeley.edu

Department of Mathematics, Evans Hall, University of California, Berkeley, CA 94720, USA 